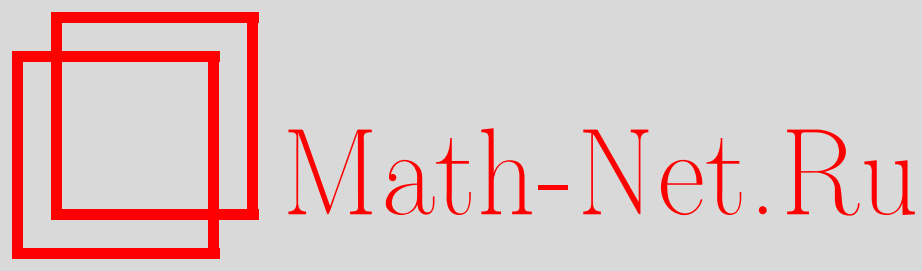

А. Ю. Хренников, Интегральная интерпретация двухчастичной волновой функции и представление квантовых корреляций с помощью случайных полей, ТМФ, 2010, том 164, номер 3, 386-393

DOI: https://doi.org/10.4213/tmf6548

Использование Общероссийского математического портала Math-Net.Ru подразумевает, что вы прочитали и согласны с пользовательским соглашением http: //www . mathnet.ru/rus/agreement

Параметры загрузки:

IP : 54.224 .135 .184

26 апреля 2023 г., 09:10:18

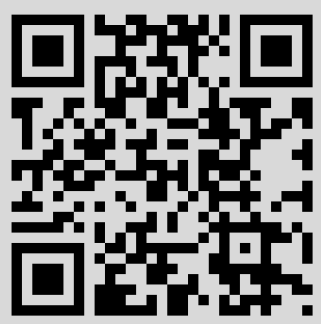




\title{
ИНТЕГРАЛЬНАЯ ИНТЕРПРЕТАЦИЯ ДВУХЧАСТИЧНОЙ ВОЛНОВОЙ ФУНКЦИИ И ПРЕДСТАВЛЕНИЕ КВАНТОВЫХ КОРРЕЛЯЦИЙ С ПОМОЩЬЮ СЛУЧАЙНЫХ ПОЛЕЙ
}

\begin{abstract}
Предложена новая интерпретация волновой функции $\Psi(x, y)$ двухчастичной квантовой системы. Волновая функция интерпретируется не как элемент функционального пространства $L_{2}$ квадратично-интегрируемых функций, т. е. вектор, а как ядро интегрального оператора (Гильберта-Шмидта). Первая часть работы посвящена выражению квантовых средних, включая корреляции в двухчастичных системах, с помощью операторной волновой функции. Это новое математическое представление в рамках традиционной квантовой механики. Однако новая интерпретация волновой функции не только порождает новый математический формализм для квантовой механики, но и позволяет выйти за ее пределы, т.е. представить квантовые корреляции (включая запутанные системы) как корреляции (гауссовых) случайных полей.
\end{abstract}

Ключевые слова: классические волны, квантовые средние, волновая функция, интегральный оператор.

\section{1. ВВЕДЕНИЕ}

Настоящая работа основана на очевидном математическом наблюдении. Любую квадратично-интегрируемую функцию $\Psi(x, y), x \in \mathbf{R}^{n_{1}}, y \in \mathbf{R}^{n_{2}}$, можно рассматривать не только как вектор, т. е. элемент пространства $L_{2}\left(\mathbf{R}^{n_{1}+n_{2}}\right)$, но и как ядро интегрального оператора $\widehat{\Psi}: L_{2}\left(\mathbf{R}^{n_{2}}\right) \rightarrow L_{2}\left(\mathbf{R}^{n_{1}}\right)$ класса Гильберта-Шмидта. В терминах этого оператора $\widehat{\Psi}$ мы находим представление корреляций в двухчастичной системе, а также средние наблюдаемых для каждой частицы. Таким образом, в зависимости от предпочтения можно использовать или векторную, или операторную интерпретацию волновой функции.

Новая интерпретация волновой функции не только порождает новый математический формализм для квантовой механики (KM), но и позволяет выйти за ее

${ }^{*}$ International Center for Mathematical Modeling in Physics, Engineering, Economics, and Cognitive Science, Linnaeus University, Växjö-Kalmar, Sweden.

E-mail: Andrei.Khrennikov@lnu.se 
пределы: представить квантовые корреляции (включая корреляции для запутанных систем) как корреляции (гауссовых) случайных полей. В работе [1] этот подход был реализован в абстрактном гильбертовом пространстве ${ }^{1)}$. Использование волновых функций, интегральных ядер и гауссовых случайных полей связывает теорию с физическим пространством (см. работы [5], [6]) и существенно проясняет связь КМ с классической теорией поля.

\section{2. ОПЕРАТОРНАЯ ИНТЕРПРЕТАЦИЯ}

2.1. Двухчастичная волновая функция как интегральное ядро. Положим $H_{1}=L_{2}\left(\mathbf{R}^{n_{1}}\right), H_{2}=L_{2}\left(\mathbf{R}^{n_{2}}\right)$ и $H=L_{2}\left(\mathbf{R}^{n_{1}+n_{2}}\right)$. Выбранное $\Psi \in H$ определяет линейный оператор $\widehat{\Psi}: H_{2} \rightarrow H_{1}$, который задается как

$$
\widehat{\Psi} \phi(x)=\int d y \Psi(x, y) \phi(y),
$$

т. е. своим ядром

$$
K_{\Psi}(x, y)=\Psi(x, y) .
$$

Это оператор типа оператора Гильберта-Шмидта. Согласно формуле (П.1) (см. приложение 1) его сопряженный оператор $\widehat{\Psi}^{*}: H_{1} \rightarrow H_{2}$ имеет ядро $K_{\Psi^{*}}(y, x)=\overline{\Psi(x, y)}$. Таким образом, $\widehat{\Psi}^{*} \phi(y)=\int d x \overline{\Psi(x, y)} \phi(x)$.

Теперь возьмем функцию $\Psi \in H$, нормированную на единицу. Согласно традиционной аксиоматике KM, она определяет чистое состояние двухчастичной системы $S=\left(S_{1}, S_{2}\right)$. Теперь представим чистое состояние системы $S=\left(S_{1}, S_{2}\right)$ как интегральный оператор. Наша цель состоит в том, чтобы выразить КМ-корреляции (для наблюдаемых на подсистемах $S_{1}$ и $S_{2}$ ), используя язык этих операторов.

2.2. Частичные следы. Найдем ядро оператора $\widehat{\Psi} \widehat{\Psi}^{*}: H_{1} \rightarrow H_{1}$. Так как

$$
\begin{aligned}
\widehat{\Psi} \widehat{\Psi}^{*} \phi(x) & =\int d y \Psi(x, y) \int d \alpha \phi(\alpha) \overline{\Psi(\alpha, y)}= \\
& =\int d y d \alpha \overline{\Psi(\alpha, y)} \Psi(x, y) \phi(\alpha)=\int d \alpha \phi(\alpha) \int d y \overline{\Psi(\alpha, y)} \Psi(x, y),
\end{aligned}
$$

то ядро имеет вид

$$
K_{\Psi \Psi^{*}}(x, \alpha)=\int d y \overline{\Psi(\alpha, y)} \Psi(x, y)
$$

Положим

$$
\hat{\rho}_{\Psi}=\Psi \otimes \Psi, \quad \hat{\rho}^{(1)}=\operatorname{Tr}_{H_{2}} \hat{\rho}_{\Psi}, \quad \hat{\rho}^{(2)}=\operatorname{Tr}_{H_{1}} \hat{\rho}_{\Psi},
$$

где $\Psi \in H$. Если $\Psi$ нормирована на единицу, то $\hat{\rho}_{\Psi}$ является оператором плотности, соответствующим чистому квантовому состоянию $\Psi$ системы $S=\left(S_{1}, S_{2}\right)$ (согласно терминологии традиционной $\mathrm{KM})$, а $\hat{\rho}^{(i)}, i=1,2$, - редуцированные операторы плотности подсистем $S_{i}$. Подчеркнем, что когда в настоящей работе мы говорим об операторной интерпретации двухчастичной волновой функции, речь идет не о стандартном соотнесении оператора плотности с любым чистым квантовым состоянием.

\footnotetext{
1) Для средних одночастичных систем, см. также работы [2]-[4].
} 
Последний действует в тензорном произведении пространств состояний $H_{i}, i=1,2$. Наше операторное представление $\Psi \mapsto \widehat{\Psi}$ позволяет обойтись без операторов, действующих в тензорном произведении.

Сравнивая выражения (1) и (П.4) (см. приложение 2), получаем равенство

$$
\widehat{\Psi} \widehat{\Psi}^{*}=\hat{\rho}^{(1)} .
$$

В отличие от вещественного случая, в комплексном случае равенство

$$
\widehat{\Psi}^{*} \widehat{\Psi}=\hat{\rho}^{(2)}
$$

не выполняется. Здесь ситуация несколько сложнее (см. п. 2.4). Прежде чем перейти к рассмотрению этой задачи, следует рассмотреть специальный класс интегральных операторов. Он сыграет важную роль в будущей теории.

Теперь возьмем квантовую наблюдаемую $\hat{A}_{1}$ (самосопряженный оператор), действующую в $H_{1}$. Имеем

$$
\operatorname{Tr} \widehat{\Psi} \widehat{\Psi}^{*} \hat{A}_{1}=\operatorname{Tr} \hat{\rho}^{(1)} \hat{A}_{1} .
$$

Это $\widehat{\Psi}$-операторное представление КМ-среднего подсистемы $S_{1}$ двухчастичной системы $S$.

2.3. Комплексное сопряжение ядра. Пусть $\hat{A}$ - интегральный оператор, для простоты симметричный. Определим оператор $\hat{\bar{A}}$ как интегральный оператор с ядром $\bar{A}(x, y)=\overline{A(x, y)}$, т. е.

$$
\hat{\bar{A}} \phi(x)=\int \overline{A(x, y)} \phi(y) d y
$$

Заметим, что $\hat{\bar{A}}$ также симметричен:

$$
\begin{aligned}
\int d x \hat{\bar{A}} \phi(x) \overline{\phi(x)} & =\int d x \int d y \overline{A(x, y)} \phi(y) \overline{\phi(x)}=\int d y \phi(y) \overline{\int d x A(x, y) \phi(x)}= \\
& =\int d y \phi(y) \overline{\int d x \overline{A(y, x)} \phi(x)}=\int d y \phi(y) \hat{\bar{A}} \phi(y) .
\end{aligned}
$$

Кроме того, заметим, что если $\hat{A} \geqslant 0$, то $\hat{\bar{A}} \geqslant 0$ :

$$
\begin{aligned}
\int d x \int d y \overline{A(x, y)} \phi(y) \overline{\phi(x)} & =\int d x \overline{\phi(x)} \overline{\int d y A(x, y) \overline{\phi(y)}}= \\
& =\int d x \overline{\phi(x)} \overline{\hat{A} \bar{\phi}(x)}=\int d x \hat{A} \bar{\phi}(x) \phi(x) \geqslant 0 .
\end{aligned}
$$

Таким образом, если $\hat{\rho}$ - оператор плотности, то $\hat{\bar{\rho}}$ - также оператор плотности.

2.4. Частичные следы. Так как

$$
\begin{aligned}
\widehat{\Psi}^{*} \widehat{\Psi} \phi(x) & =\int d u \widehat{\Psi} \phi(u) \overline{\Psi(u, x)}= \\
& =\int d u \int d v \phi(v) \Psi(u, v) \overline{\Psi(u, x)}=\int d v \phi(v) \int d u \Psi(u, v) \overline{\Psi(u, x)}
\end{aligned}
$$


то ядро имеет вид

$$
K_{\Psi^{*} \Psi}(x, v)=\int d u \Psi(u, v) \overline{\Psi(u, x)} .
$$

Используя формулу (П.6) (см. приложение 2), получаем равенство $\overline{K_{\rho^{(2)}}\left(x_{2}, y_{2}\right)}=$ $K_{\Psi^{*} \Psi}\left(x_{2}, y_{2}\right)$. Таким образом, в отличие от равенства $\hat{\rho}^{(1)}=\widehat{\Psi} \widehat{\Psi}^{*}$ получаем

$$
\widehat{\bar{\rho}^{(2)}}=\widehat{\Psi}^{*} \widehat{\Psi} .
$$

Теперь возьмем квантовую наблюдаемую $\hat{A}_{2}$ (самосопряженный оператор), действующую в $H_{2}$. Используя формулу (П.7) (см. приложение 3 ), получаем

$$
\operatorname{Tr} \widehat{\Psi}^{*} \widehat{\Psi} \hat{A}_{2}=\operatorname{Tr} \hat{\rho}^{(2)} \hat{A}_{2} .
$$

Это $\widehat{\Psi}$-операторное представление КМ-среднего подсистемы $S_{2}$ двухчастичной системы $S$.

2.5. Корреляции. Возьмем две квантовые наблюдаемые: $\hat{A}_{1}$, которая действует в $H_{1}$, и $\hat{A}_{2}$, которая действует в $H_{2}$ (самосопряженные операторы). Рассмотрим следующую композицию операторов:

$$
\begin{aligned}
\widehat{M} \phi(x) & =\widehat{\Psi} \hat{\bar{A}}_{2} \widehat{\Psi}^{*} \hat{A}_{1} \phi(x)=\int d y \Psi(x, y) \hat{\bar{A}}_{2} \widehat{\Psi}^{*} \hat{A}_{1} \phi(y)= \\
& =\int d y \Psi(x, y) \int d \alpha \overline{A_{2}(y, \alpha)} \widehat{\Psi}^{*} \hat{A}_{1} \phi(\alpha)= \\
& =\int d y d \alpha \Psi(x, y) \overline{A_{2}(y, x)} \int d \beta \overline{\Psi(\beta, \alpha)} \hat{A}_{1} \phi(\beta)= \\
& =\int d y d \alpha d \beta d \gamma \Psi(x, y) \overline{\Psi(\beta, \alpha)} A_{1}(\beta, \gamma) \overline{A_{2}(y, \alpha)} \phi(\gamma)
\end{aligned}
$$

Отсюда

$$
K_{M}(x, u)=\int d y d \alpha d \beta \Psi(x, y) \overline{\Psi(\beta, \alpha)} A_{1}(\beta, u) \overline{A_{2}(y, \alpha)}
$$

поэтому

$$
\operatorname{Tr} \widehat{M}=\int d x K_{M}(x, x)=\int d x d y d \alpha d \beta \Psi(x, y) \overline{\Psi(\beta, \alpha)} A_{1}(\beta, x) A_{2}(\alpha, y)
$$

Теперь рассмотрим оператор $\hat{A}=\hat{A}_{1} \otimes \hat{A}_{2}: H \rightarrow H$. Он представляет собой квантовую наблюдаемую для двухчастичной системы $S=\left(S_{1}, S_{2}\right)$, описывающую корреляции между наблюдаемыми $\hat{A}_{i}, i=1,2$, для подсистем $S_{i}$. Найдем его КМ-среднее (используя традиционную векторную интерпретацию волновой функции):

$$
\begin{aligned}
\hat{A} \Psi\left(x_{1}, x_{2}\right) & =\int d y_{1} d y_{2} A_{1} \otimes A_{2}\left(x_{1}, x_{2}, y_{1}, y_{2}\right) \Psi\left(y_{1}, y_{2}\right)= \\
& =\int d y_{1} d y_{2} A_{1}\left(x_{1}, y_{1}\right) A_{2}\left(x_{2}, y_{2}\right) \Psi\left(y_{1}, y_{2}\right) .
\end{aligned}
$$

Получаем

$$
\langle\hat{A}\rangle_{\Psi} \equiv\langle\hat{A} \Psi, \Psi\rangle=\int d x_{1} d x_{2} d y_{1} d y_{2} A_{1}\left(x_{1}, y_{1}\right) A_{2}\left(x_{2}, y_{2}\right) \Psi\left(y_{1}, y_{2}\right) \overline{\Psi\left(x_{1}, x_{2}\right)}
$$


В равенстве (6) положим $\beta \rightarrow x_{1}, x \rightarrow y_{1}, \alpha \rightarrow x_{2}, y \rightarrow y_{2}$. Получаем

$$
\operatorname{Tr} \widehat{M}=\int d x_{1} d x_{2} d y_{1} d y_{2} A_{1}\left(x_{1}, y_{1}\right) A_{2}\left(x_{2}, y_{2}\right) \Psi\left(y_{1}, y_{2}\right) \overline{\Psi\left(x_{1}, x_{2}\right)} .
$$

Таким образом, квантовую корреляцию можно представить с использованием

$$
\langle\hat{A}\rangle_{\Psi}=\operatorname{Tr} \widehat{\Psi} \hat{\bar{A}}_{2} \widehat{\Psi}^{*} \hat{A}_{1}
$$

\section{3. ПРЕДКВАНТОВАЯ КЛАССИЧЕСКАЯ СТАТИСТИЧЕСКАЯ ТЕОРИЯ ПОЛЯ}

\section{1. Представление квантовых корреляций в терминах случайных по-} лей. В ряде работ (см., например, [1], [4]) мы пропагандировали предквантовую модель, в которой роль скрытых параметров играют классические поля. Подробности этого подхода, так называемой предквантовой классической статистической теории поля (ПКСТП), можно найти в упомянутых работах. Предквантовая переменная, соответствующая квантовой наблюдаемой $\hat{A}$, задается квадратичной формой

$$
f_{A}(\phi)=\int A(x, y) \phi(x) \overline{\phi(y)} d x d y .
$$

Рассмотрим группу $G_{2}=\{e, *\}$, где $*^{2}=e$. Она естественным образом действует в пространстве $L_{2}$ комплекснозначных функций: $\phi \rightarrow \bar{\phi}$. Возьмем некоторое пространство, обозначаемое как $F\left(L_{2}, \mathbf{R}\right)$, (вещественнозначных) функционалов $f$ : $L_{2} \rightarrow \mathbf{R}$. Действие группы $G_{2}$ в $L_{2}$ можно поднять на пространство $F\left(L_{2}, \mathbf{R}\right): f \rightarrow \bar{f}$, где $\bar{f}(\phi)=f(\bar{\phi})$.

Сравним квадратичные формы операторов $\hat{A}$ и $\hat{\bar{A}}$ :

$$
\begin{aligned}
f_{\bar{A}}(\phi) & =\int \overline{A(x, y)} \phi(y) \overline{\phi(x)} d x d y=\int A(y, x) \phi(y) \overline{\phi(x)} d x d y= \\
& =\int A(x, y) \phi(x) \overline{\phi(y)} d x d y=f_{A}(\bar{\phi}) .
\end{aligned}
$$

Рассмотрим векторное гауссово случайное поле $\omega \rightarrow \phi(x ; \omega)=\left(\phi_{1}(x ; \omega), \phi_{2}(x ; \omega)\right)$ с нулевым средним. Возьмем две квантовые наблюдаемые $\hat{A}_{i}, i=1,2$, на подсистемах $S_{i}$ двухчастичной системы $S=\left(S_{1}, S_{2}\right)$ и соответствующие предквантовые квадратичные функционалы $f_{A_{1}}\left(\phi_{1}\right), f_{A_{2}}\left(\phi_{2}\right)$. Подставляя компоненты $\phi(x ; \omega)$ в эти функционалы, получим квадратичные функционалы гауссовых случайных полей. Теперь возьмем такое гауссово случайное поле, что корреляция между его компонентами задается оператором $\widehat{\Psi}$, т. е. формально для любых двух точек $x_{0}, y_{0} \in \mathbf{R}^{3}$ выполнено

$$
E \phi_{1}\left(x_{0} ; \omega\right) \phi_{2}\left(y_{0} ; \omega\right)=\Psi\left(x_{0}, y_{0}\right) .
$$

Мы не будем вдаваться в математические подробности построения этого случайного поля (см. [1]). Одна из математических сложностей заключается в том, что его компоненты нельзя выбрать в виде функций из $L_{2}$. Необходимо рассмотреть расширение Гильберта-Шмидта пространства $L_{2}$. Согласно работе [1] имеем равенство

$$
E f_{A_{1}} \bar{f}_{A_{2}}=E f_{A_{1}} E \bar{f}_{A_{2}}+\operatorname{Tr} \widehat{\Psi} \hat{\bar{A}}_{2} \widehat{\Psi}^{*} \hat{A}_{1},
$$


где $E$ обозначает среднее по (классическому) гауссовому случайному полю. Это равенство доказывается прямым вычислением среднего от произведения двух квадратичных форм гауссовых случайных полей.

Наконец, применяя (7), получаем представление квантовой корреляции в виде дисперсии двух классических случайных переменных, квадратичных функционалов гауссовых случайных полей:

$$
\operatorname{cov}\left(f_{A_{1}}, \bar{f}_{A_{2}}\right)=\left\langle\hat{A}_{1} \otimes \hat{A}_{2}\right\rangle_{\Psi}
$$

3.2. К экспериментальной проверке предсказаний ПКСТП. Равенство (9) показывает, что корреляции традиционных квантовых наблюдаемых можно выразить в терминах классических случайных полей. Однако ПКСТП не только воспроизводит предсказания КМ, с ее помощью можно получить и ряд новых предсказаний. С помощью ПКСТП можно измерить (по крайней мере в принципе) не только квадратичные функционалы предквантового поля, но и функционалы любого типа. Неквадратичные функционалы не имеют аналогов в традиционной КМ. Они представляют новый класс наблюдаемых, так сказать, предквантовые наблюдаемъе. Простейший класс таких наблюдаемых задается линейными функционалами предквантовых полей. Они представляют собой сглаживания компонент этих полей. Если пренебречь необходимостью сглаживания, то ПКСТП предсказывает корреляцию между компонентами “двухчастичного предквантового случайного поля" в двух различных точках, которая задается равенством (8). По-видимому, это равенство дает правильную физическую интерпретацию двухчастичной волновой функции.

ПРИЛОЖЕНИЕ 1

\section{Сопряженный оператор и след интегрального оператора}

Напомним несколько равенств для ядра интегрального оператора. Начнем с замечания по поводу сопряженного оператора:

$$
\int d x \int d y A(x, y) \phi_{1}(y) \overline{\phi_{2}(x)}=\int d y \phi_{1}(y) \overline{\int d x \overline{A(x, y)} \phi_{2}(x)} .
$$

Таким образом, $\hat{A}^{*}$ имеет ядро $A^{*}(\alpha, \beta)=\overline{A(\beta, \alpha)}$; в частности, $\hat{A}$ является самосопряженным, если

$$
A(\alpha, \beta)=\overline{A(\beta, \alpha)}
$$

Теперь напомним интегральное представление следа. Будем действовать формально. Чтобы наше рассмотрение было совсем строгим, следует воспользоваться теорией обобщенных функций. Возьмем ортонормированный базис в $L_{2}$, скажем, $\left\{\phi_{k}\right\}$. Тогда для любого $f \in L_{2}$ имеем

$$
f(x)=\sum_{k} \int f(y) \overline{\phi_{k}(y)} d y \phi_{k}(x)=\int d y f(y) \sum_{k} \phi_{k}(x) \overline{\phi_{k}(y)} .
$$


Таким образом (по крайней мере формально), $\sum_{k} \phi_{k}(x) \overline{\phi_{k}(y)}=\delta(x-y)$. Теперь рассмотрим интегральный оператор $\hat{A}$ и найдем его след:

$$
\begin{aligned}
\left.\sum_{k} \int d x \int d y A(x, y) \phi_{k}(y)\right) \overline{\phi_{k}(x)} & =\int d x d y A(x, y) \sum_{k} \phi_{k}(y) \overline{\phi_{k}(x)}= \\
& =\int d x d y \delta(x-y) A(x, y) .
\end{aligned}
$$

Получаем

$$
\operatorname{Tr} \hat{A}=\int d x A(x, x)
$$

\section{ПРИЛОЖЕНИЕ 2}

\section{Ядра частичных следов}

Пусть теперь $\hat{A}$ действует в $H$ :

$$
\hat{A} \phi\left(x_{1}, x_{2}\right)=\int d y_{1} d y_{2} A\left(x_{1}, x_{2}, y_{1}, y_{2}\right) \phi\left(y_{1}, y_{2}\right) .
$$

Найдем интегральное представление его частичного следа по $H_{2}$. Для этого рассмотрим $x_{1}, y_{1}$ как параметры. Таким образом, находим ядро оператора $\operatorname{Tr}_{H_{2}} \hat{A}$ :

$$
A_{H_{2}}\left(x_{1}, y_{1}\right)=\int d z A\left(x_{1}, z, y_{1}, z\right)
$$

Теперь найдем ядро $H_{2}$-следа проекционного оператора $\hat{\rho}_{\Psi}=\Psi \otimes \Psi, \Psi \in H$. Имеем

$$
\hat{\rho}_{\Psi} u\left(x_{1}, x_{2}\right)=\int d \alpha d \beta \overline{\Psi(\alpha, \beta)} u(\alpha, \beta) \Psi\left(x_{1}, x_{2}\right) .
$$

Таким образом, ядро имеет вид

$$
K_{\rho_{\Psi}}\left(x_{1}, x_{2}, y_{1}, y_{2}\right)=\Psi\left(x_{1}, x_{2}\right) \overline{\Psi\left(y_{1}, y_{2}\right)} .
$$

Оператор частичного $H_{2}$-следа $\hat{\rho}^{(1)}=\operatorname{Tr}_{H_{2}} \hat{\rho}_{\Psi}$ имеет ядро

$$
K_{\rho^{(1)}}\left(x_{1}, y_{1}\right)=\int d z \Psi\left(x_{1}, z\right) \overline{\Psi\left(y_{1}, z\right)}
$$

и действует как

$$
\hat{\rho}^{(1)} g(x)=\int d y K_{\rho^{(1)}}(x, y) g(y)=\int d y d z \Psi(x, z) \overline{\Psi(y, z)} g(y) .
$$

Найдем ядро оператора $\hat{\rho}^{(2)}=\operatorname{Tr}_{H_{1}} \hat{\rho}_{\Psi}$ :

$$
K_{\rho^{(2)}}\left(x_{2}, y_{2}\right)=\int d z \Psi\left(z, x_{2}\right) \overline{\Psi\left(z, y_{2}\right)}
$$


ПРИЛОЖЕНИЕ 3

\section{Одно равенство для следов}

Рассмотрим произвольный оператор плотности $\hat{\rho}$ и квантовую наблюдаемую $\hat{A}$, а также операторы, получаемые посредством комплексного сопряжения их ядер. Будем использовать равенство

$$
\operatorname{Tr} \hat{\bar{\rho}} \hat{\bar{A}}=\operatorname{Tr} \hat{\rho} \hat{A} \text {. }
$$

Докажем его: $K_{\bar{\rho} \bar{A}}(x, y)=\int d u \overline{\rho(x, u)} \overline{A(u, y)}$, а потому

$$
\operatorname{Tr} \hat{\bar{\rho}} \hat{\bar{A}}=\int d x d u \overline{\rho(x, u)} \overline{A(u, x)}=\int d x d u \rho(u, x) A(x, u)=\operatorname{Tr} \hat{\rho} \hat{A} .
$$

\section{Список литературы}

[1] A. Khrennikov, Demystification of quantum entanglement, arXiv: 0905.4791.

[2] A. Bach, J. Math. Phys., 14:1 (1981), 125-132; Phys. Lett. A, 73:4 (1979), 287-288; J. Math. Phys., 21:4 (1980), 789-793.

[3] J. Kupsch, O. G. Smolyanov, N. A. Sidorova, J. Math. Phys., 42:3 (2001), 1026-1037, arXiv: math-ph/0012025.

[4] A. Khrennikov, J. Math. Phys., 48:1 (2007), 013512, 18 pp.; J. Phys. A, 38:41 (2005), 9051-9073, arXiv: quant-ph/0505228; Found. Phys. Lett., 18:7 (2005), 637-650; Phys. Lett. A, 357:3 (2006), 171-176, arXiv: quant-ph/0602210; Found. Phys. Lett., 19:4 (2006), 299-319; Nuovo Cimento B, 121:5 (2006), 505-521, arXiv: hep-th/0604163; Phys. Lett. A, 372:44 (2008), 6588-6592.

[5] I. V. Volovich, "Quantum cryptography in space and Bell's theorem", Foundations of Probability and Physics, Quantum Probab. White Noise Anal., 13, ed. A. Khrennikov, World Sci., River Edge, NJ, 2001, 364-372.

[6] I. V. Volovich, "Towards quantum information theory in space and time", Quantum Theory: Reconsideration of Foundations, ed. A. Khrennikov, Växjö Univ. Press, Växjö, 2002, 423-440. 